\title{
Analysis and optimization of DC supply range for the ESP32 development board
}

\author{
Joshua Kim
}

\begin{abstract}
ESP32 is becoming a popular and potential game-changer in the IoT industry. Once a code completed, to takeoff out of a USB power, questions rise about powering it. What's the feasible external voltage range? What's the current? Which cell battery? And so on. These questions cannot be easily resolved by only skimming datasheets.

This paper went over to clarify the obscure information about the DC supply range for the ESP32 development board, especially ESP 32-DevKitC V4. The results were disclosed through investigation, calculation, experiments, and LTspice simulation. Starting from getting relevance facts from datasheets of essential components on the board, calculated thermal conditions of heated component, experimented to confirm the calculated and get practical data while code running including GPIO and $\mathrm{WiFi}$, and ended with simulation to confirm the data.

This paper concludes the following result points. The minimum supply voltage is $3.6 \mathrm{~V}$ to run an ESP32 module. The supply voltage should be under $10 \mathrm{~V}$ for both input capacitor rated voltage and LDO junction temperature rating. The thermal restriction was calculated at an ambient temperature of $25^{\circ} \mathrm{C}$ and tried and tested. For a more harsh environment, the upper limit voltage could be derated in this way. An external power should be able to supply current well over average $100 \mathrm{~mA}$; a good $1 \mathrm{~A}$. In terms of battery, this range reassures that an ESP32 can run with a single cell LiPo. Regarding the USB, both the high power and low power port can supply sufficiently. While an external supply being no less than $5.2 \mathrm{~V}$, both the USB and external sources could work simultaneously.
\end{abstract}

\section{Index Terms}

ESP32, Internet of things (IoT), Embedded systems, Microcontrollers, Thermal management of electronics

\section{INTRODUCTION}

$\mathbf{E}$ SPRESSIF's ESP32 microcontroller is becoming popular in the embedded and IoT industry due to its many pros [1]. Its dual-core speeds up and FreeRTOS support attracts more and more developers [2]. Also, Espressif provides the ESP32 module and AWS ${ }^{1}$ certified development board ESP 32-DevKitC $\mathrm{V} 4$ at an affordable price for makers or developers to get easily started even on a breadboard. Moreover, one can write the code on the programming environment ESP-IDF (Espressif IoT Development Framework) or even with Arduino IDE, which makes diverse kinds of users could handle it from high school science club to experienced industry experts.

To power the development board, there are two options; USB or EXT_5V pin on the connector J2 as shown in Fig. 1. This paper primarily focuses on using the $\mathrm{EXT} \_5 \mathrm{~V}$ pin. Another way of giving $3.3 \mathrm{~V}$ directly to the $3.3 \mathrm{~V}$ pin is beyond the scope of this paper.

The EXT_5V pin doesn't have to be exact $5.0 \mathrm{~V}$ due to no $5 \mathrm{~V}$ user component on the board except for the LDO, U2 AMS1117-3.3 [4]. The U1 CP2102 (USB to UART bridge) gets the USB VBUS (5 V from USB).

Regarding using the EXT_5V, there could happen a controversy or fallacy for anyone who has little time to ponder the hardware. To get the correct decision, this paper introduces a reliable supply range to the ESP32-DevKitC V4 or compatibles out of both intuitive and quantitative analysis.

\footnotetext{
joshua.kim@ieee.org

${ }^{1}$ Amazon Web Services
} 


\section{APPROACH}

\section{A. Components Electrical Characteristics}

Table I shows the electrical characteristics of the essential components in Fig. 1 [4][5][6][7].

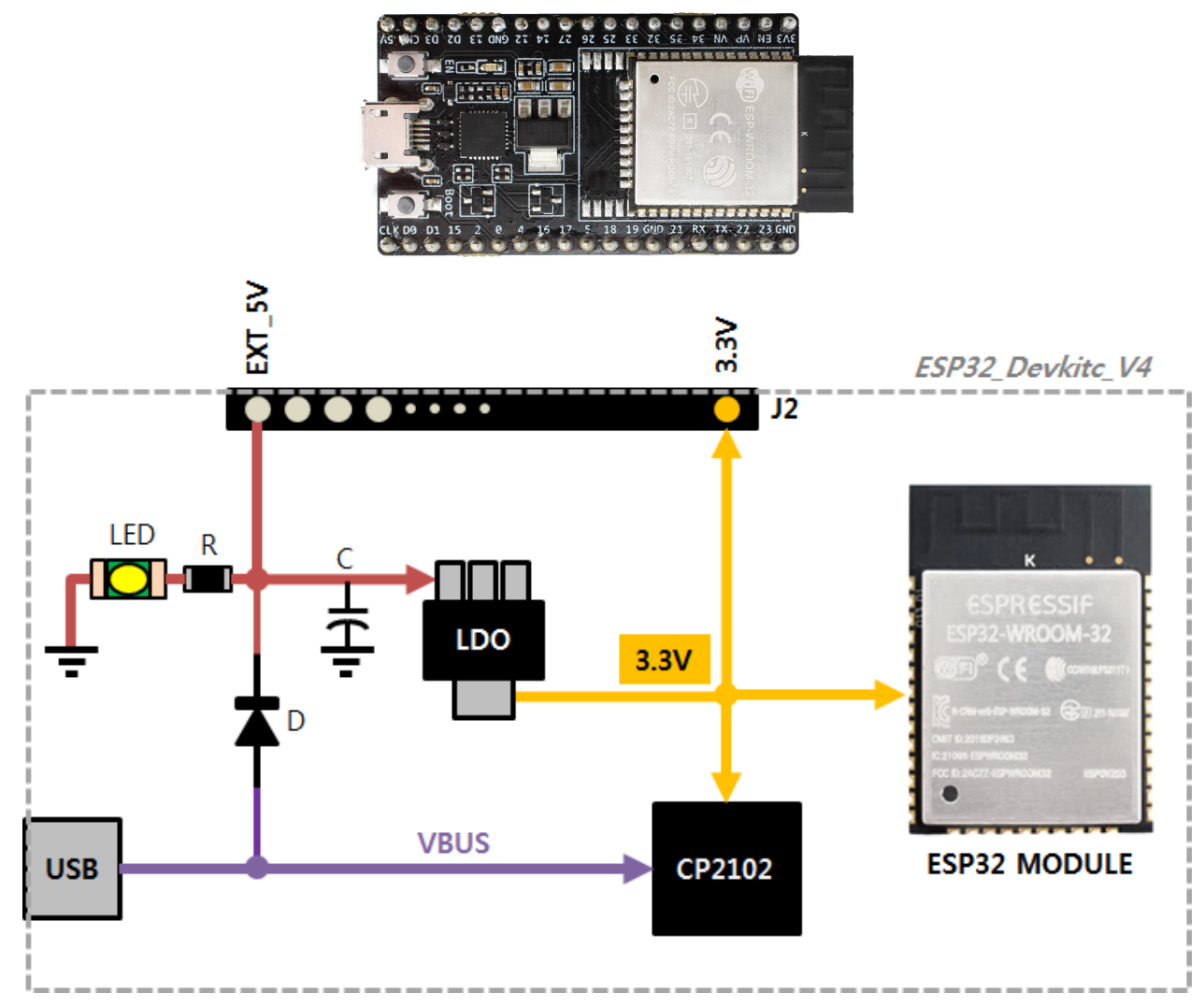

Fig. 1. ESP32 development board and its simplified power path out of ESP32-DevKitC V4 circuit diagram

TABLE I

ESSENTIAL CHARACTERISTICS OF COMPONENTS ON THE VEXT_5V OR V $3.3 \mathrm{~V}$ NETS

\begin{tabular}{|c|c|c|c|c|}
\hline & $\mathrm{C}$ & LDO & $\mathrm{CP} 2102$ & ESP32 \\
\hline \hline$V_{\max }$ & $10 \mathrm{~V}$ & $15 \mathrm{~V}$ & $3.6 \mathrm{~V}$ & $3.6 \mathrm{~V}$ \\
\hline$V_{\min }$ & & & $3.0 \mathrm{~V}$ & $2.3 \mathrm{~V}$ \\
\hline$I_{\text {norm }}$ & & & $20 \mathrm{~mA}$ & $80 \mathrm{~mA}$ \\
\hline$I_{P U}$ & & & $0.2 \mathrm{~mA}$ & \\
\hline$I_{\text {Lim }}$ & & $900 \mathrm{~mA}$ & & \\
\hline$P_{D \max }$ & & $1.2 \mathrm{~W}$ & & \\
\hline
\end{tabular}

$\mathrm{V}_{\text {EXT_5V }}$, the supplied voltage to $\mathrm{J} 2 \mathrm{EXT} \_5 \mathrm{~V}$ pin, covers through $\mathrm{C}, 22 \mu \mathrm{F} / 10 \mathrm{~V}$ ceramic capacitor, and input of LDO which downward regulates to $3.3 \mathrm{~V}$ for the ESP32 module and CP2012. The three constraints are as follows:

1) The minimum $V_{\text {EXT_5V }}$ has to satisfy for the LDO to supply $V_{\text {min }}$ to both CP2102 and ESP32. Let $\mathrm{V}_{\text {EXT_5V }}$ and $\mathrm{V}_{3.3 \mathrm{~V}}$ denote the input range to the LDO and corresponding output region respectively, a function $f$ maps the two spaces; $f: \mathrm{V}_{\mathrm{EXT}_{-} 5 \mathrm{~V}} \rightarrow \mathrm{V}_{3.3 \mathrm{~V}}$

$$
f(x) \geq \max \left\{V_{\min C P 2012}, V_{\min E S P 32}\right\}, x \in \mathrm{V}_{\mathrm{EXT}_{-} 5 \mathrm{~V}}
$$

2) $\mathrm{V}_{\text {EXT_5V }}$ should be less than the capacitor $\mathrm{C}$ rated voltage, $10 \mathrm{~V}$.

$$
\mathrm{V}_{\text {EXT_5V }}<10 \mathrm{~V}
$$


3) The LDO confines power dissipation at most $1.2 \mathrm{~W}$ to guarantee its line and load regulation [6]. Where $P_{D}$ is the power dissipation of LDO, U2 AMS1117-3.3,

$$
P_{D} \leq 1.2 \mathrm{~W}
$$

\section{B. Minimum Input Voltage}

To satisfy Equation (1), $f(x)$ looks to have to be greater than $V_{\min C P 2012}$ in Table I. However, CP2012 only works while coding phase binary update or console diagnostics under USB supplied. Once USB disconnected, CP2012 consumes $I_{P U} 200 \mu \mathrm{A}$ while do actually nothing. So ESP32's $V_{\min }$ is crucial as long as supplied from EXT_5V. ESP32's datasheet shows its $V_{\min }=2.3 \mathrm{~V}$ and average current $I_{\text {norm }}=80 \mathrm{~mA}$ [5]. To confirm these terms two experiments carried out.

- The first experiment outputted GPIO PWM at frequency $300 \mathrm{kHz}$ and duty swept from 0 to $100 \%$ every second with no RF.

- The latter ran a web server that treats three query packets a second.

For the experiment, BK precision 9206 bench power supplied at 2 A current limit with 18 AWG $80 \mathrm{~cm}$ stranded wire to the development board. Fluke 8846A measured average current $I_{\text {norm }}$ with Analyze function.

As far as the board boot and run the codes, $\mathrm{V}_{\text {EXT_5V }}$ was adjusted to the minimum, where $\mathrm{V}_{3.3 \mathrm{~V}}$ and $I_{\text {norm }}$ were recorded and $P_{D}$ was calculated accordingly in Table II.

TABLE II

Minimum Voltage EXPeriment Result

\begin{tabular}{|c|c|c|c|c|c|}
\hline Experiment & $\mathrm{WiFi}$ & $\mathrm{V}_{\text {EXT_5V }}$ & $\mathrm{V}_{3.3 \mathrm{~V}}$ & $I_{\text {norm }}$ & $P_{D}$ \\
\hline \hline 1. LED PWM (300 kHz) & $\mathrm{X}$ & $3.5 \mathrm{~V}$ & $2.5 \mathrm{~V}$ & $42 \mathrm{~mA}$ & $0.042 \mathrm{~W}$ \\
\hline 2. Web Server & $\mathrm{O}$ & $3.6 \mathrm{~V}$ & $2.6 \mathrm{~V}$ & $100 \mathrm{~mA}$ & $0.100 \mathrm{~W}$ \\
\hline
\end{tabular}

\section{Maximum Input Voltage}

Under the conditions (2) and (3), the maximum $\mathrm{V}_{\text {EXT_5V }}$ is bounded by LDO's junction temperature out of power dissipation determined by the following equation.

$$
P_{D}=\frac{T_{j}-T_{a}}{\theta_{j a}}
$$

$$
\begin{aligned}
P_{D} & =\text { power dissipation in watts } \\
T_{j} & =\text { maximum junction temperature } \\
T_{a} & =\text { operating temperature } \\
\theta_{j a} & =\text { thermal resistance from the device junction to ambient }
\end{aligned}
$$

The LDO AMS1117-3.3 datasheet shows that $T_{j}$ is rated at $125{ }^{\circ} \mathrm{C}, \theta_{j a}$ is $90{ }^{\circ} \mathrm{C} / \mathrm{W}$ at an ambient temperature of $T_{a}=25^{\circ} \mathrm{C}$ [6]. Little copper pad beneath the LDO on the development board retains the $\theta_{j a}$ underrated [3]. Substituting these numbers into Equation (4) gives maximum $P_{D}=$ which is in this case less than 1.1 watts.

$$
P_{D}<\frac{125^{\circ} \mathrm{C}-25^{\circ} \mathrm{C}}{90^{\circ} \mathrm{C} / \mathrm{W}}=1.1 \mathrm{~W}
$$

The LDO's power dissipation has to be less than $1.1 \mathrm{~W}$, which is included in the constraint (3). Applying the current $100 \mathrm{~mA}$ from Table II, the maximum $\mathrm{V}_{\mathrm{EXT}_{-} 5 \mathrm{~V}}$ could be calculated. However, the result exceeds the capacitor rated voltage (2), which remains dominant. 
To validate the condition (2) meets the thermal limitation (5), the same codes in section II-B ran with $\mathrm{V}_{\text {EXT_5V }}=9 \mathrm{~V}$. Additionally measured was case temperature $T_{c}$ converging over running 6 hours in a $25{ }^{\circ} \mathrm{C}$ air-conditioned room. Omega UWBT thermometer captured the $T_{c}$ with a thermocouple on the LDO tab.

TABLE III

$\mathrm{V}_{\text {EXT_5V }}=9$ V EXPERIMENT RESULT

\begin{tabular}{|c|c|c|c|c|c|}
\hline Experiment & $\mathrm{WiFi}$ & $T_{c}$ & $\mathrm{~V}_{3.3 \mathrm{~V}}$ & $I_{\text {norm }}$ & $P_{D}$ \\
\hline \hline 1. LED PWM (300 kHz) & $\mathrm{X}$ & $40^{\circ} \mathrm{C}$ & $3.3 \mathrm{~V}$ & $46 \mathrm{~mA}$ & $0.26 \mathrm{~W}$ \\
\hline 2. Web Server & $\mathrm{O}$ & $54{ }^{\circ} \mathrm{C}$ & $3.3 \mathrm{~V}$ & $100 \mathrm{~mA}$ & $0.57 \mathrm{~W}$ \\
\hline
\end{tabular}

III. RESULTS

From Table II and III, the Equations (1) and (2) result simplified to

$$
2.6 \mathrm{~V} \leq f\left(x \in \mathrm{V}_{\mathrm{EXT}_{-} 5 \mathrm{~V}}\right) \leq 3.3 \mathrm{~V}, \mathrm{~V}_{\mathrm{EXT}_{-} 5 \mathrm{~V}}:=\{x \in \mathbb{R} \mid 3.6 \leq x<10\} \mathrm{V}
$$

At least $\mathrm{V}_{\text {EXT_5V }} 3.6 \mathrm{~V}$ assures the minimum $\mathrm{V}_{3.3 \mathrm{~V}} 2.6 \mathrm{~V}$ for $\mathrm{RF}$ applications can work in order.

Table III shows that the condition (2) complies with the LDO's thermal restriction. From the measured $T_{c}$ and $T_{a}$, the junction temperature can be estimated far below the rated $T_{j}$. We conclude the available $\mathrm{V}_{\mathrm{EXT} \_5 \mathrm{~V}}$ range.

$$
\therefore 3.6 \mathrm{~V} \leq \mathrm{V}_{\text {EXT_5V }}<10 \mathrm{~V}
$$

\section{DISCUSSION}

\section{A. Simulation}

An LTspice circuit simulation reassured the result condition (7). Advanced Monolithic Systems, the AMS1117 manufacturer, doesn't provide its SPICE model. So a very similar LT1117-3.3 library acted the LDO [9]. The input voltage swept from $3 \mathrm{~V}$ to $9 \mathrm{~V}$ linearly in the simulation circuit Fig. 2. As a load, a current source sunk $50 \mathrm{~mA}, 100 \mathrm{~mA}$, and $200 \mathrm{~mA}$ respectively.

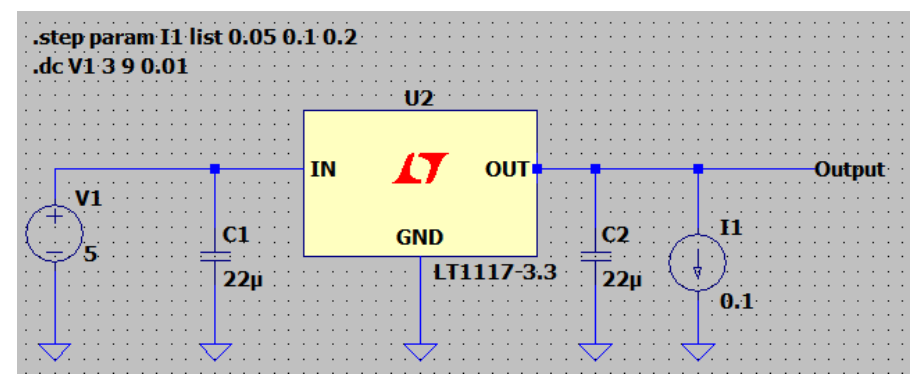

Fig. 2. Simulation circuit for LDO response to varying input. Capacitor C1 and C2 have the same value as in the ESP32-DevKitC V4 circuit.

The simulation outcome in Fig. 3 shows that the input $3.6 \mathrm{~V}$ makes output $2.6 \mathrm{~V}$, which is exactly the experiment result in Table II. The LDO response is consistent regardless of the load current variance. 


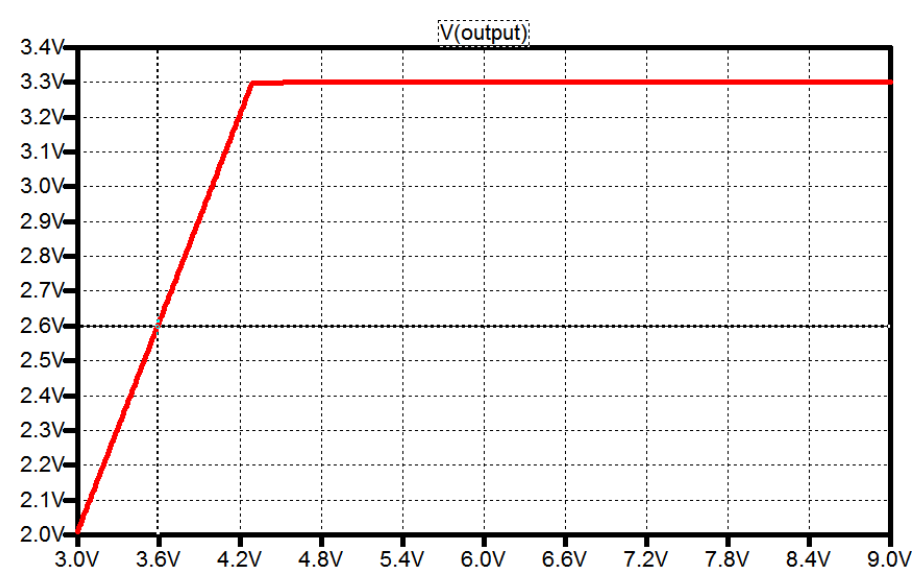

Fig. 3. Simulation result. The horizontal axis $\equiv$ input voltage, vertical axis $\equiv$ output voltage. LDO makes $2.6 \mathrm{~V}$ at input $3.6 \mathrm{~V}$.

\section{B. USB}

USB is prevalent to be used with no problem. The USB VBUS ranges from $4.4 \mathrm{~V} \sim 5.5 \mathrm{~V}$ at Lowpower Port, and $4.7 \mathrm{~V} \sim 5.5 \mathrm{~V}$ at High-power Port [8]. Where $V_{f}$ is the diode D BAT760-7 forward voltage drop max $0.5 \mathrm{~V}$ [10], the worst-case LDO input is

$$
\begin{array}{ll}
\text { VBUS }_{\text {min }}-V_{f(\max )}=4.4 \mathrm{~V}-0.5 \mathrm{~V}=3.9 \mathrm{~V} & \text { (Low-power Port) } \\
\text { VBUS }_{\text {min }}-V_{f(\max )}=4.7 \mathrm{~V}-0.5 \mathrm{~V}=4.2 \mathrm{~V} & \text { (High-power Port) }
\end{array}
$$

Either the USB Low-power port or High-power port sufficiently meets Equation (7), for the LDO to supply ESP32 sufficiently.

\section{Battery-powered system}

A single-cell LiPo battery that covers $3.7 \mathrm{~V} \sim 4.2 \mathrm{~V}$ confirms the requirement (7). It can directly supply the ESP32 development board.

\section{Dual power source at the same time}

Could the USB power and EXT_5V be used simultaneously? In Fig. 1, a diode D BAT760-7 exists between the VBUS and EXT_5V [4]. It has forward voltage drop $V_{f}=0.3 \mathrm{~V} \sim 0.5 \mathrm{~V}$ [10]. Now that

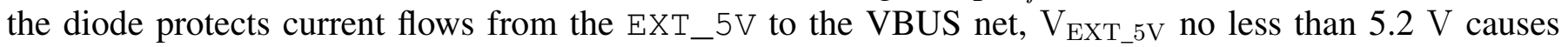
no problem while the USB lives at the same time.

$$
\begin{aligned}
\text { VBUS }_{\text {max }}-V_{f(\text { min })} & \leq \mathrm{V}_{\text {EXT_5V }}<10 \mathrm{~V} \\
5.2 \mathrm{~V} & \leq \mathrm{V}_{\mathrm{EXT}_{-} 5 \mathrm{~V}}<10 \mathrm{~V}
\end{aligned}
$$

\section{Conclusions}

This paper deciphers pertinent datasheets and papers to disclose and clarify primary facts as follows for designing the power supply chain for the ESP32 module and reassured them with experiment and simulation. 
It would be better to go for the $V_{\min }$ value a bit higher than that of the ESP32 datasheet which must be measured in an ideal laboratory environment; At least $2.6 \mathrm{~V}$ is recommended to run an EPS32 module especially for an RF application from an experiment.

This paper suggests considering the average current consuming $100 \mathrm{~mA}$ while RF works busily.

At least $3.6 \mathrm{~V}$ and under $10 \mathrm{~V}$, the available external supply voltage $\mathrm{V}_{\text {EXT_5V }}$ range introduced. The lower limit is for the ESP32's $V_{\min }$. The higher bound concerns both the input capacitor rated voltage and LDO's rated junction temperature. Even though the thermal condition was calculated and tested at an ambient temperature of $25^{\circ} \mathrm{C}$, one could refine and derate the upper limit voltage in this way for a more harsh environments.

Both the USB and EXT_5V could work simultaneously while the $\mathrm{V}_{\text {EXT_5V }}$ condition (8) meets.

The ESP32-DevKitC V4 can be said flawless for powered by either a single cell battery or USB.

\section{REFERENCES}

[1] A. Maier, A. Sharp and Y. Vagapov, "Comparative analysis and practical implementation of the ESP32 microcontroller module for the internet of things," 2017 Internet Technologies and Applications (ITA), Wrexham, 2017, pp. 143-148, doi: 10.1109/ITECHA.2017.8101926.

[2] K. Dokic, M. Martinovic and B. Radisic, "Neural Networks with ESP32 - Are Two Heads Faster than One?," 2020 6th Conference on Data Science and Machine Learning Applications (CDMA), Riyadh, Saudi Arabia, 2020, pp. 141-145, doi: 10.1109/CDMA47397.2020.00030.

[3] D. Hollander, "Packaging trends and mounting techniques for power surface mount components," Proceedings of 1995 International Conference on Power Electronics and Drive Systems. PEDS 95, Singapore, 1995, pp. 264-270 vol.1, doi: 10.1109/PEDS.1995.404911.

[4] Espressif, https://dl.espressif.com/d1/schematics/esp32_devkitc_v4-sch.pdf, 2017.

[5] Espressif Systems, https://www.espressif.com/sites/default/files/documentation/esp32_datasheet_en.pdf, 2020.

[6] Advanced Monolithic Syatems, http://www.advanced-monolithic.com/pdf/ds1117.pdf

[7] Silicon Labs, https://www.silabs.com/documents/public/data-sheets/CP2102-9.pdf, 2017.

[8] USB Implementers Forum, https://www.usb.org/document-library/usb-20-specification, 2019.

[9] Analog Devices, https://www.analog.com/media/en/technical-documentation/data-sheets/1117fd.pdf

[10] Diodes, https://www.diodes.com/assets/Datasheets/ds30498.pdf, 2017. 